University of Nebraska - Lincoln

DigitalCommons@University of Nebraska - Lincoln

2012

\title{
The structure of posttraumatic psychopathology in veterans attending primary care
}

\author{
David Forbes \\ University of Melbourne \\ Jon D. Elhai \\ University of Toledo \\ Emma Lockwood \\ University of Melbourne \\ Mark Creamer \\ University of Melbourne \\ B. Christopher Frueh \\ University of Hawaii
}

See next page for additional authors

Follow this and additional works at: https://digitalcommons.unl.edu/publichealthresources

Part of the Public Health Commons

Forbes, David; Elhai, Jon D.; Lockwood, Emma; Creamer, Mark; Frueh, B. Christopher; and Magruder, Kathryn M., "The structure of posttraumatic psychopathology in veterans attending primary care" (2012). Public Health Resources. 171.

https://digitalcommons.unl.edu/publichealthresources/171

This Article is brought to you for free and open access by the Public Health Resources at DigitalCommons@University of Nebraska - Lincoln. It has been accepted for inclusion in Public Health Resources by an authorized administrator of DigitalCommons@University of Nebraska - Lincoln. 


\section{Authors}

David Forbes, Jon D. Elhai, Emma Lockwood, Mark Creamer, B. Christopher Frueh, and Kathryn M. Magruder 


\title{
The structure of posttraumatic psychopathology in veterans attending primary
} care $^{\text {זे }}$

\author{
David Forbes $^{\mathrm{a}, *}$, Jon D. Elhai ${ }^{\mathrm{b}}$, Emma Lockwood $^{\mathrm{a}}$, Mark Creamer ${ }^{\mathrm{a}}$, B. Christopher Frueh $^{\mathrm{c}, \mathrm{d}}$, \\ Kathryn M. Magruder ${ }^{\mathrm{e}}$
}

a Australian Centre for Posttraumatic Mental Health and Department of Psychiatry, University of Melbourne, Level 1/340 Albert St., East Melbourne, Victoria 3002, Australia
b Department of Psychology, University of Toledo, Toledo, OH, USA
${ }^{\mathrm{c}}$ Department of Psychology, University of Hawaii, Hilo, HI, USA
${ }^{\mathrm{d}}$ The Menninger Clinic, Houston, TX, USA
${ }^{\mathrm{e}}$ Veterans Administration Medical Center and Department of Psychiatry and Behavioral Sciences, Medical University of South Carolina, Charleston, SC, USA

\section{A R T I C L E I N F O}

\section{Article history:}

Received 25 May 2011

Received in revised form

20 September 2011

Accepted 24 September 2011

\section{Keywords:}

Posttraumatic stress disorder

Veterans

Latent factors

Anxiety

Mood

\begin{abstract}
A B S T R A C T
This study attempted to extend research indicating that posttraumatic stress disorder (PTSD) factors of Re-experiencing, Avoidance and Hyperarousal are more related to Fear/phobic disorders, while PTSD Dysphoria is more related to Anxious-Misery disorders. Trauma exposure, PTSD and comorbidity data for 668 veteran patients were analysed using confirmatory factor analyses and relative strengths of the relationships between PTSD factors and the Fear and Anxious-Misery factors were assessed. Combining Simms, Watson, and Doebbeling's (2002) model of PTSD symptoms and Krueger's (1999) Fear/Anxious Misery model of mood and anxiety disorders fit the data well. Contrary to previous research, PTSD Reexperiencing, Avoidance and Hyperarousal did not correlate more with the Fear factor; nor did PTSD Dysphoria correlate more with Anxious-Misery. Hyperarousal was more closely related to Fear than was Re-experiencing; however, Avoidance was not. Dysphoria was more closely related to the Anxious-Misery factor than all other PTSD factors.
\end{abstract}

(c) 2011 Elsevier Ltd. All rights reserved.
Posttraumatic stress disorder (PTSD) as currently defined has high rates of comorbidity with other mood and anxiety disorders (Creamer, Burgess, \& McFarlane, 2001; Kessler, Sonnega, Bromet, Hughes, \& Nelson, 1995) and a heterogeneous clinical presentation (Blanchard, Hickling, Taylor, Loos, \& Gerardi, 1994), features which are thought to pose a challenge to its diagnostic validity (Frueh, Elhai, \& Acierno, 2010; McNally, 2003; Rosen \& Lilienfeld, 2008; Spitzer, First, \& Wakefield, 2007). Research exploring the structural relationship between PTSD and other mood and anxiety disorders (e.g. Brown \& McNiff, 2009; Elhai, Carvalho, Palmieri, Primi, \& Frueh, 2011; Forbes et al., 2010; Frueh et al., 2000; Grant, Beck, Marques, Palyo, \& Clapp, 2008; Gros, Simms, \& Acierno, 2010; Simms, Watson, \& Doebbeling, 2002) has begun to shed light on the nature and origins of this comorbidity and heterogeneity. Forbes et al. (2010) found that, in a sample of acute traumatic injury survivors, the PTSD symptoms more specific to the disorder (Reexperiencing, Avoidance and Hyperarousal) were more related to a Fear or phobic disorders factor underlying mood and anxiety disor-

\footnotetext{
is All authors had full access to all of the data in the study and take responsibility for the integrity of the data and the accuracy of the data analysis.

* Corresponding author. Tel.: +61 39936 5100; fax: +61 399365199.

E-mail address: dforbes@unimelb.edu.au (D. Forbes).
}

ders (Krueger, 1999), while the PTSD dysphoria symptoms (Simms et al., 2002) were more closely related to the Anxious-Misery factor (generalized anxiety disorder and major depression; Grant et al., 2008). This study aims to replicate Forbes et al.'s (2010) findings in a veterans' general medical patient sample. It also seeks to extend these findings by clarifying differences between the PTSD factors in terms of their relationship to each of the Fear and AnxiousMisery factors. This will help improve diagnostic specificity as well as treatment selection for varying presentations of PTSD symptoms.

In addition to exposure to a traumatic event, DSM-IV diagnostic criteria for PTSD require at least 1 of 5 re-experiencing symptoms, at least 3 of 7 avoidance and numbing symptoms, and at least 2 of 5 arousal symptoms (American Psychiatric Association, 1994). However, confirmatory factor analyses (CFAs) suggest that PTSD is better represented by a four-factor model, either one of two very similar models specified by King, Leskin, King and Weathers (1998) and Simms et al. (2002) respectively. Simms et al.'s (2002) four-factor model comprises a Re-experiencing factor (DSM-IV PTSD items B1-B5), an Avoidance factor (C1-C2), a Dysphoria factor (C3-C7; D1-D3) and a Hyperarousal factor (D4-D5). Simms et al.'s (2002) Dysphoria factor comprises items such as diminished interest (C4), restricted affect (C6), difficulty sleeping (D1), irritability (D2) and poor concentration (D3), which are also symptoms of major depressive disorder (MDD) and/or generalized 
anxiety disorder (GAD). This Dysphoria factor may thus reflect the non-specific general distress or negative affectivity component identified by the structural models of Clark and Watson (1991) and Brown, Chorpita, and Barlow (1998) as common to many mood and anxiety disorders. The presence of dysphoria symptoms in the diagnostic criteria for PTSD might therefore account for high rates of comorbidity between PTSD and other mood and anxiety disorders and its heterogeneous clinical presentation (Forbes et al., 2010; Simms et al., 2002). Consistent with this, empirical analyses by Grant et al. (2008) found considerable shared variance between this Dysphoria factor and GAD and MDD, while the other three factors of Simms et al.'s (2002) model were more specific to PTSD.

Recent research (e.g. Cox, Clara, \& Inns, 2002; Forbes et al., 2010; Slade \& Watson, 2006) has attempted to locate PTSD within, or relate its factor structure to, the structure of mood and anxiety disorders overall. One influential approach to modelling the structure of mood and anxiety disorders was developed by Krueger (1999), who identified two latent factors underlying mood and anxiety disorders, Fear and Anxious-Misery. Disorders loading onto the Fear factor included social phobia, simple phobia, agoraphobia, obsessive-compulsive disorder and panic disorder, while MDD, dysthymia and GAD loaded onto the Anxious-Misery factor. PTSD was not included in Krueger's (1999) analysis, but subsequent studies (e.g. Cox et al., 2002; Slade \& Watson, 2006) found that PTSD loaded onto the Anxious-Misery factor. This is inconsistent with the conceptualization of PTSD as a fear-based disorder (Creamer, Burgess, \& Pattison, 1992; Foa, Steketee, \& Rothbaum, 1989) associated with persisting autonomic arousal (Brown \& McNiff, 2009) and phobic responses to trauma, and which is responsive to prolonged exposure therapy (Keane \& Kaloupek, 1982), a conceptualization supported by biological and psychophysiological studies (Pitman, Orr, Forgue, de Jong, \& Claiborn, 1987).

Why, then, would PTSD have correlated more with the AnxiousMisery (rather than Fear) factor in Cox et al.'s (2002) and Slade and Watson's (2006) studies? Forbes et al. (2010) suggested that the inclusion of Simms et al.'s (2002) dysphoria symptoms (i.e. criteria C3-D3) in the DSM-IV criteria for PTSD may explain this finding. Slade and Watson's (2006) finding that the correlation between PTSD and Anxious-Misery decreased from .83 to .69 when ICD10 criteria, which include fewer dysphoria symptoms, were used also supports this hypothesis. The re-experiencing, active avoidance and hyperarousal symptoms of Simms et al.'s (2002) model, in contrast, have been shown to be more specific to PTSD (Grant et al., 2008), are clinically and theoretically associated with fear, and thus may be more associated with the Fear factor.

Cox et al. (2002) and Slade and Watson (2006) treated PTSD as a single entity that could load onto either the Fear and Anxious-Misery factors. However, the hypothesized association of PTSD's Dysphoria factor with the Anxious-Misery factor and PTSD's remaining three factors with the Fear factor suggest that it would be useful to disaggregate PTSD into its constituent factors and test their relationships with the Fear and Anxious-Misery factors separately. Accordingly, Forbes et al. (2010), collected PTSD symptom and mood and anxiety disorder diagnosis data from a sample of 714 physical injury survivors fitting them to a model that specified mood and anxiety disorders to load onto Fear and Anxious-Misery factors (Krueger, 1999) and PTSD symptoms onto a four-factor model (Simms et al., 2002). The Fear and Anxious-Misery factors were allowed to correlate with each of the four PTSD symptom factors. Fit indices for the combined model indicated excellent fit, and the Re-experiencing, active Avoidance and Hyperarousal factors were significantly more correlated with the Fear factor than with the Anxious-Misery factor, while the Dysphoria factor was significantly more correlated with the Anxious-Misery factor.

Forbes et al.'s (2010) findings held for three different time points post-trauma: 3 months, 12 months and 24 months. However, the sample had experienced only a subset of possible PTSD criterion A events, specifically traffic accidents, falls, assaults, and other injuries necessitating hospital treatment. To ensure their generalizability, these results need to be replicated in broader samples, such as those encountered in general medical settings. Testing this research question with primary care patients provides a wider range of PTSD severity than the more severe end found in specialty mental health patients. In addition, sequelae from a vast array of traumatic events, including interpersonal trauma such as combat, assault and sexual assault, are treated in primary care. Primary care is the fastest growing sector of mental healthcare delivery (Wang et al., 2006), and typically acts as the gate-keeper for provision of mental health services in both Veterans Administration and other healthcare sectors. Evidence suggests that people often prefer discussing their mental health problems with primary care professionals (Del Piccolo, Saltini, \& Zimmerman, 1998), and this preference is highlighted in military populations where there is a significant stigma about visiting mental health professionals (Hoge et al., 2004).

Replicating Forbes et al.'s (2010) study would also provide an opportunity to further examine PTSD symptom factors and the relationship to the Anxious-Misery and Fear factors. For example, it is important to examine the extent to which the PTSD symptom factors may differ in the strength of their relationships with the Anxious-Misery and Fear factors. Avoidance symptoms (items C1-C2) of PTSD may be particularly Fear-related in that they constitute a typically phobic response and hyperarousal symptoms (Simms et al., 2002) such as hypervigilance and exaggerated startle response may also be Fear-related, reflecting excessive autonomic arousal similar to that seen in panic disorder. Re-experiencing however, appears to comprise a more heterogeneous set of symptoms that may reduce the strength of its relationship with the Fear factor. Recurrent distressing dreams (criterion B2) may be accurate replays of traumatic experiences that awaken the dreamer "in fright". Alternatively, they may be more symbolic in content (Phelps, Forbes \& Creamer, 2008) involving emotions such as anger, guilt and general distress, which may awaken the dreamer, but not necessarily "in fright". Similarly, DSM-IV does not require that the intense psychological distress at exposure to internal or external cues symbolizing the trauma (criterion B4) be of a specifically fearful nature. Thus, these two symptoms and the Re-experiencing factor more generally, may be less strongly correlated with the Fear factor than the Avoidance and Hyperarousal factors. Given its overlap with symptoms of MDD and GAD, the Dysphoria factor should be significantly more correlated with the Anxious-Misery factor than the other three PTSD factors.

This study therefore aims to replicate Forbes et al.'s (2010) findings in a veterans primary care medical sample, hypothesizing that (1) the four factor PTSD model (consistent with Simms et al., 2002) combined with the two factor Fear and Anxious Misery model for the anxiety and depressive disorders fits the data well; (2) the PTSD specific factors of Re-experiencing, Avoidance and Hyperarousal (D4-5) are more correlated with Fear than Anxious Misery, and the PTSD Dysphoria factor is more highly correlated with Anxious Misery than Fear. This study also seeks to extend these previous findings with a further hypothesis that (3) Avoidance and Hyperarousal are more closely related to Fear than is Re-experiencing and Dysphoria is more closely related to Anxious Misery than are the other 3 PTSD factors.

\section{Method}

\subsection{Procedures}

Data were derived from a cross-sectional survey conducted on a random sample of veterans at four VA Medical Centers' 
primary care clinics in the Southeastern U.S. (Charleston and Columbia, SC; Tuscaloosa and Birmingham, AL). Participants were randomly selected from a master list of eligible patients (determined as discussed below). Participants who provided written informed consent (after being given a complete study description) were administered a semi-structured clinic assessment, and within two months were administered a structured telephone interview by masters-level clinicians, trained and supervised by a licensed clinical psychologist $(\mathrm{BCF})$. All phone interviews were initiated from the Charleston VA. Initial exclusionary criteria included dementia-related symptoms (based solely on medical recordsbased diagnoses) and being age 80 or older; ineligible participants were not randomly selected for initial research contact. The study was conducted with full approval from relevant Institutional Review Boards; a full procedural description can be found elsewhere (Magruder et al., 2005).

\subsection{Participants}

A total of 1198 randomly identified veterans were approached for study participation; nearly three-quarters ( $n=885,74 \%$ ) provided informed consent. Of this subset, 747 veterans were available for a telephone interview.

Of the 747 participants, 696 (93.2\%) were men. Age averaged 61.2 years $(S D=11.8)$. The majority were Caucasian $(n=468,62.7 \%)$ or African American $(n=259,34.7 \%)$. One-third of participants were employed ( $n=246,32.9 \%) ; 281$ (37.6\%) were retired, and 220 (29.5\%) were disabled. About half of participants ( $n=379,50.7 \%)$ reported attaining no more than a high school education, while 249 (33.3\%) reported some college education, and 119 (15.9\%) graduated college. Nearly half of participants $(n=371,49.7 \%)$ served in a war zone. Based on the measures administered (below), 90 participants (12.0\%) met criteria for PTSD.

\subsection{Measures}

In addition to collecting sociodemographic data via self-report and medical records, measures relevant to the present paper are described below. The CAPS and MINI were administered and scored by trained clinicians, supervised by a licensed psychologist (BCF).

\subsubsection{Trauma Assessment for Adults-Self Report Version (TAA)}

The TAA (Resnick, Best, Kilpatrick, Freedy, \& Falsetti, 1993) is a brief measure designed to assess for a lifetime history of PTSD's criterion A traumatic events. Using behaviorally specific questions, the TAA includes queries for 13 traumatic events, including follow-up questions of traumatic event-related characteristics. The TAA demonstrates moderate to substantial temporal stability, and responses substantially converge with those from other validated trauma exposure measures in clinical and non-clinical samples (Gray, Elhai, Owen, \& Monroe, 2009).

\subsubsection{Clinician-Administered PTSD Scale (CAPS)}

The CAPS (Blake et al., 1995) is a structured clinical interview designed for rating the frequency and intensity of PTSD's 17 DSMIV symptoms. After a given symptom is presented, respondents are asked to rate the symptom separately based on its frequency and intensity, using behaviorally precise rating anchors (on a "0"-“4" scale). Thus 34 queries are used to assess PTSD's 17 symptoms. The CAPS is the most widely used interviewer-administered instrument for PTSD diagnostic assessment (Elhai, Gray, Kashdan, \& Franklin, 2005). Across studies, the CAPS has demonstrated high inter-rater reliability ( $r$ s of .90 or higher), high internal consistency (alphas $=.80-.90)$, and convergent validity with other PTSD measures ( $r s=.70$ or higher) (reviewed in Weathers, Keane, \& Davidson, 2001 ). In the present dataset, inter-rater reliability evidenced $100 \%$ concordance based on a random sample of $8 \%$ of CAPS interviews (Magruder et al., 2005), and internal consistency was .98 for both the frequency and intensity item formats. Several studies have supported the four-factor Dysphoria PTSD model as fitting well using the CAPS (Forbes et al., 2010; Palmieri, Weathers, Difede, \& King, 2007). The present study assessed current PTSD symptoms, based on the respondent's self-nominated most distressing traumatic event from the TAA.

\subsubsection{Mini International Neuropsychiatric Interview (MINI)}

The MINI (Sheehan et al., 1998) is a brief structured diagnostic interview developed for DSM-IV and ICD-10 mental disorders. Inter-rater reliability is reported to be excellent, with most kappa values being .90 or higher and none below .75 . Adequate test-retest reliability is reported, with most kappa values above .75 , and only one (current manic episode) below .45. The MINI demonstrates good concordance with the Structured Clinical Interview for DSM-IV's Axis I disorders (SCID), with good or very good kappa values, and only a single value (current drug dependence) below .50 (Sheehan et al., 1998). Sensitivity is reported to be .70 or greater for all but three disorders (dysthymia, obsessive-compulsive disorder, and current drug-dependence), with specificity of .85 or higher across all diagnoses (Sheehan et al., 1998, 1997). We used version 5.0.0, using only current (as opposed to lifetime) MINI diagnoses.

\subsection{Analyses}

We excluded participants who did not endorse at least one traumatic event and were thus not administered the CAPS ( $n=78)$, and one participant who was not administered the MINI, leaving an effective sample size of 668 . Given the nature of the intervieweradministered diagnostic interviewing, no missing CAPS items were present; only 21 subjects were missing MINI diagnostic variables (1-2 variables each). All analyses were conducted using Mplus 6 software (Muthén \& Muthén, 2010), with missing MINI data modelled using maximum likelihood procedures with a pairwise present estimation approach by using all individuals with observations on a pair of variables (Muthén \& Muthén, 1998-2010).

We first modelled a CFA based on Simms et al.'s (2002) fourfactor model. We summed each PTSD symptom's frequency and intensity ratings to result in 17 CAPS PTSD items. These PTSD items evidenced considerable non-normality, with 10 items evidencing skewness $>2$ and four items with kurtosis $>6$. The CFA therefore used maximum likelihood estimation with a mean-adjusted Satorra-Bentler chi-square statistic, robust to non-normality (Satorra \& Bentler, 2001). Each factor's last unstandardized factor loading was fixed to 1 for model identification, and residual error covariances were fixed to zero. Goodness of fit indices are reported, including the comparative fit index (CFI), Tucker-Lewis index (TLI), root mean square error of approximation (RMSEA), and standardized root mean square residual (SRMR). Models that fit very well (or adequately) are indicated by CFI and TLI $\geq .95$ (.90-.94), RMSEA $<.06$ (to .08), and SRMR <.08 (to .10) (Hu \& Bentler, 1999).

Next, we used CFA to model the Fear and Anxious Misery disorders. We defined the Fear factor using panic disorder (with or without agoraphobia), agoraphobia without a history of panic disorder, obsessive compulsive disorder, and social phobia, with social phobia's unstandardized loading fixed to 1 . We defined the Anxious Misery factor using major depressive episode, dysthymic disorder and generalized anxiety disorder, with major depressive episode's unstandardized loading fixed to 1 . Since the diagnostic variables were binary ("present"|“absent") ratings, we implemented weighted least squares estimation with a meanand variance-adjusted chi-square (WLSMV) for this CFA, using 


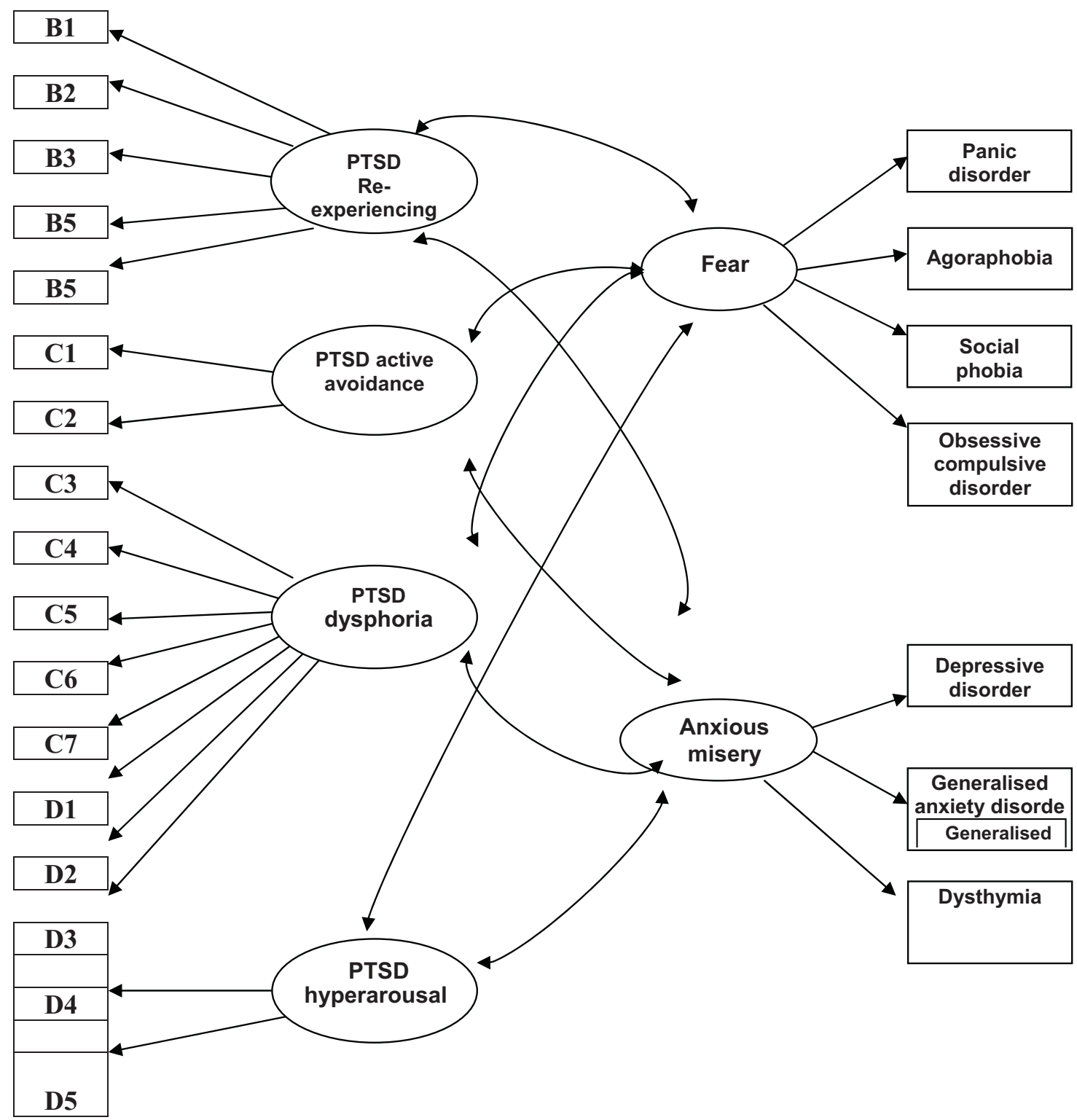

Fig. 1. Structure of the model comprising a four-latent factor structure for PTSD and two latent factors of common mental disorders, Fear and Anxious Misery.

tetrachoric (rather than Pearson) correlations and probit (rather than linear) regression coefficients (Flora \& Curran, 2004; Wirth \& Edwards, 2007). Residual error covariances were fixed to zero. Goodness of fit indices are reported as above, but not SRMR since it is not sensitive to good model fit when using categorical variables (Yu, 2002).

We then tested the full structural equation model, integrating the two CFAs and allowing each CFA's latent factors to correlate with the other CFA's latent factors. Given the presence of binary variables, we used WLSMV estimation (see Fig. 1).

We used Wald chi-square tests to evaluate whether there was a significant difference between each PTSD factor's correlation with the Fear factor and the Anxious-Misery factor. We also used Wald chi-square tests to evaluate in a pairwise fashion whether there was a significant difference between the correlation of the PTSD Dysphoria factor with the Anxious-Misery factor compared to the correlations between the other PTSD factors and the AnxiousMisery factor. Finally, we used Wald chi-square tests to evaluate in a pair-wise fashion differences between the PTSD Re-experiencing, Avoidance and Hyperarousal factors in the strengths of their correlation with the Fear factor.

\section{Results}

The rates of exposure to the various events assessed on the TAA (Resnick et al., 1993) are presented in Table 1.

CAPS total scores ranged from 0 to $119(M=14.50, S D=27.44)$. Prevalence of PTSD on the CAPS was $12 \%$. Prevalence of MINI disorders included $17.8 \%$ (major depressive episode), $13.9 \%$ (dysthymia), 13.1\% (generalized anxiety disorder), 8.0\% (panic disorder), 3.9\% (social phobia), 2.1\% (obsessive compulsive disorder), and $1.2 \%$ (agoraphobia without panic).

A CFA for the four-factor PTSD model yielded excellent model fit, S-B $\chi^{2}(113, N=668)=222.94, p<.001, \mathrm{CFI}=.97, \mathrm{TLI}=.97$, RMSEA $=.04$, SRMR $=.02$. Uniformly strong standardized factor loadings of greater than .85 were present (except for PTSD's item reflecting psychogenic amnesia, as expected).

A CFA for the two-factor disorders model (Fear and AnxiousMisery) yielded excellent model fit, robust $\chi^{2}(13, N=668)=46.09$, $p<.001, \mathrm{CFI}=.97, \mathrm{TLI}=.95, \mathrm{RMSEA}=.06$. Standardized factor loadings were greater than .75 , except for that of agoraphobia.

The structural equation model, integrating the two measurement models (CFAs) and the two models' factors allowed 
Table 1

Rates of participants' exposure to events assessed by the Trauma Assessment for Adults-Self Report Version (TAA), $n=668$.

\begin{tabular}{lr}
\hline Event & $\%$ \\
\hline War zone/military combat & 54.9 \\
Pre World War II & .9 \\
World War II & 14.2 \\
Pre Korean War & 1.5 \\
Korean War & 10.6 \\
Pre Vietnam War & 1.5 \\
Vietnam War & 25.6 \\
Post Vietnam War & 1.2 \\
1991 Gulf War & 3.9 \\
Serious accident & 39.5 \\
Sexual contact with adult prior to age of 13 & 4.9 \\
Sexual assault prior to age of 18 & 2.2 \\
Sexual assault after the age of 18 & 2.5 \\
Attacked with a weapon & 20.5 \\
Attacked with a weapon with intent to kill or seriously injure & 9.6 \\
Other physical injury & 7.9 \\
Other threat of death or serious injury & 15.3 \\
Witnessed others killed or seriously injured & 39.8 \\
Friend or family member killed by another person & 16.0 \\
Friend or family member killed in accident & 29.2 \\
Friend or family member killed by sudden illness & 40.1 \\
\hline
\end{tabular}

Note: Percentages do not add up to $100 \%$ as more than one event may be endorsed.

to correlate, demonstrated excellent model fit, $\chi^{2}(237$, $N=668)=340.51, p<.001, \mathrm{CFI}=.97, \mathrm{TLI}=.96, \mathrm{RMSEA}=.03$. Table 2 demonstrates the two CFA models' factor loadings.

The first set of Wald chi-square tests indicated that no PTSD factor yielded correlations that were significantly different between the mental disorders factors (see Table 3 ). The second set of Wald chi-square tests found that the Dysphoria factor was significantly more correlated with Anxious-Misery than were the

Table 2

Standardized factor loadings for the structural equation model.

\begin{tabular}{|c|c|}
\hline PTSD item & Factor loading \\
\hline \multicolumn{2}{|c|}{ PTSD's measurement model } \\
\hline \multicolumn{2}{|c|}{ PTSD's reexperiencing } \\
\hline PTSD B1 & .94 \\
\hline PTSD B2 & .92 \\
\hline PTSD B3 & .86 \\
\hline PTSD B4 & .95 \\
\hline PTSD B5 & .91 \\
\hline \multicolumn{2}{|l|}{ PTSD's Avoidance } \\
\hline PTSD C1 & .96 \\
\hline PTSD C2 & .96 \\
\hline \multicolumn{2}{|l|}{ PTSD’s Dysphoria } \\
\hline PTSD C3 & .64 \\
\hline PTSD C4 & .91 \\
\hline PTSD C5 & .91 \\
\hline PTSD C6 & .91 \\
\hline PTSD C7 & .84 \\
\hline PTSD D1 & .91 \\
\hline PTSD D2 & .88 \\
\hline PTSD D3 & .92 \\
\hline \multicolumn{2}{|c|}{ PTSD’s Hyperarousal } \\
\hline PTSD D4 & .86 \\
\hline PTSD D5 & .90 \\
\hline \multicolumn{2}{|c|}{ Fear and Anxious Misery disorder measurement model } \\
\hline \multicolumn{2}{|c|}{ Fear } \\
\hline Social phobia & .80 \\
\hline Agoraphobia & .36 \\
\hline Panic & .84 \\
\hline OCD & .77 \\
\hline \multicolumn{2}{|l|}{ Misery } \\
\hline MDE & .92 \\
\hline Dysthymia & .86 \\
\hline GAD & .70 \\
\hline
\end{tabular}

Note: OCD, obsessive compulsive disorder; MDE, major depressive episode; GAD, generalized anxiety disorder.
Table 3

Correlation coefficients for PTSD's four-factor model compared across the Fear and Anxious Misery factors.

\begin{tabular}{llll}
\hline \multirow{2}{*}{ PTSD factor } & \multicolumn{2}{l}{ Correlation with disorder factor } & Wald $\chi^{2}$ test $(d f)$ \\
\cline { 2 - 3 } & Fear & Anxious Misery & \\
\hline Reexperiencing & .56 & .57 & $.08(1)$ \\
Avoidance & .58 & .57 & $.11(1)$ \\
Dysphoria & .63 & .66 & $.43(1)$ \\
Hyperarousal & .64 & .62 & $.28(1)$ \\
\hline
\end{tabular}

Re-experiencing or Avoidance. The Dysphoria factor demonstrated a trend $(p<.08)$ toward a higher correlation with Anxious Misery than Hyperarousal did. The Fear factor was more highly correlated with the Hyperarousal PTSD factor than the Re-experiencing PTSD factor. There was no significant difference between the correlations of the Avoidance PTSD factor with the Fear factor and the Re-experiencing PTSD factor and the Fear factor. These latter results are shown in Table 4.

\section{Discussion}

The results of this paper found that the Simms et al.'s (2002) four-factor model of PTSD and a model encompassing common anxiety and mood disorders within two factors of Fear and AnxiousMisery consistent with Krueger's (1999) model fit the data well. Fit statistics remained excellent when these two measurement models were combined together. These results replicate the findings of Forbes et al. (2010) in a general veterans' medical sample. Contrary to those previous findings the three more specific PTSD factors of Re-experiencing (B1-5), Avoidance (C1-2) or Hyperarousal (D4-5) did not correlate more closely with Fear than Anxious-Misery nor did the PTSD Dysphoria factor correlate more closely with the Anxious-Misery factor. There was, however, variation between the PTSD factors in terms of their proximity to the Fear and Anxious Misery factors. As hypothesized, Hyperarousal was more closely related to Fear than was Re-experiencing. Avoidance, however, was not more closely related to Fear than Re-experiencing. Given the phobic nature of avoidance symptoms this was contrary to expectations. Interestingly there was a trend toward a significant difference between Hyperarousal and Avoidance $(p<.06)$ in their relationship with the Fear factor. Consistent with expectations, Dysphoria was more closely related to Anxious-Misery than all three other more PTSD-specific factors, although the difference with Hyperarousal was not significant $(p<.08)$.

The results of this study support variations across the four PTSD factors in the strength of their respective relationships with the Fear and Anxious-Misery factors - the strongest of these appears to be Hyperarousal's relationship with Fear and Dysphoria's relationship with Anxious-Misery and Fear. The results, however, did not support the findings of Forbes et al. (2010) of a more proximate relationship between the PTSD-specific clusters and Fear and the Dysphoria cluster and Anxious-Misery. These findings are consistent with those of others (Frueh et al., 2000; Marshall, Schell, \& Miles, 2010; Miller et al., 2010) who found that all PTSD symptoms were highly associated with measures of general distress.

In understanding the difference in findings between this study and that of Forbes et al. (2010), it may be relevant to consider sample characteristics. Forbes et al. (2010) examined a sample of traumatically injured participants in the relatively acute phase through to 2 years post-injury. In the current study, not only did the combat veterans comprising half the sample serve in conflicts dating back a number of decades (World War II, Korea and Vietnam), but also participants had experienced a range of other traumatic events, such as assault and sexual assault, including molestation during childhood. Accordingly, while precise data are lacking, the 
Table 4

Pairwise correlations between PTSD factors and Fear and Anxious-Misery factors.

\begin{tabular}{|c|c|c|c|c|c|}
\hline Re-experiencing & Avoidance & Dysphoria & Arousal & Wald $\chi^{2}$ test $(d f)$ & $p$ \\
\hline \multicolumn{6}{|c|}{ Correlations with Fear factor } \\
\hline .56 & .58 & & & $.68(1)$ & .41 \\
\hline \multirow[t]{2}{*}{.56} & & & .64 & $8.44(1)^{* *}$ & .004 \\
\hline & .58 & & .64 & $3.59(1)$ & .06 \\
\hline \multicolumn{6}{|c|}{ Correlations with Anxious-Misery factor } \\
\hline & & .66 & 62 & $2.98(1)$ & .08 \\
\hline & .57 & .66 & & $12.89(1)^{* * *}$ & .0003 \\
\hline .57 & & .66 & & $13.65(1)^{* * *}$ & .0002 \\
\hline
\end{tabular}

${ }_{* * *}^{* *} p<.01$

$p<.001$.

results may be partially accounted for by the chronic nature of the symptoms experienced by a significant portion of the sample. While more acute PTSD reactions (even up to two years) have distinctive latent structures, this distinctiveness may dissipate as the condition becomes more chronic and associated with increased amount of comorbidity and impairment in function. It is also possible that variations in the nature of the trauma may account for some of the differences in these two studies. In the mixed medical sample in the current study, exposures included both interpersonal and non-interpersonal traumas as well as both repeated and single incident traumas, with data indicating variations in symptom profiles on the basis of these traumatic events (Chung \& Breslau, 2008; Forbes et al., in press). Finally, it is likely that there are important contextual differences between a sample of civilians with acute injury and combat veterans treated in the U.S. VA healthcare system, including the availability of disability benefits and eligibility for services for those diagnosed with PTSD (Angrist, Chen, \& Frandsen, 2010; Frueh, Grubaugh, Elhai, \& Buckley, 2007).

\subsection{Limitations}

There is a range of limitations to the interpretations of the findings of this study. First, the time since the traumatic event or duration of the veterans' symptoms were not able to be clearly identified. As such, interpretations about the chronicity of the sample need to be inferred for the combat veterans from the conflicts in which they served and little comment can be made about the chronicity of the half of the sample who had not served in war zones. A further limitation is that the findings are limited to a veteran sample and results cannot be confidently generalized to other exposed populations.

\subsection{Conclusion}

This research established that a four factor model of PTSD and a two factor model covering the anxiety and mood disorders fit the data well. The research found variations across the PTSD clusters in the strength of their relationships with these two overarching Fear and Anxious Misery factors, although challenged previously identified differences in the differential relationship across Fear and Misery. Future research should continue to investigate the nature of PTSD and its relationship with broader factors underpinning anxiety and depression in order to ensure as best as possible that moving into DSM-V and beyond PTSD the unique and more specific elements of the diagnosis are not lost or overridden by shared elements that reduce diagnostic specificity and impair clinical management. The current proposed revisions to DSM-V, now being tested in field trials in the U.S., likely represent a modest improvement over the DSM-IV criteria, though they are incremental and relatively minor in nature (Frueh, Elhai, \& Acierno, 2010). Thus, they are unlikely to adequately address the concerns highlighted by these results.

\section{Conflict of interest}

The Australian Centre for Posttraumatic Mental Health is partially funded by the Australian Government Department of Veterans' Affairs.

\section{Acknowledgements}

This study was supported by grants VCR-99-010-2 from Veterans Affairs Health Services Research and Development (Veterans Affairs HSR\&D) to K.M.M. and an Australian National Health and Medical Research Council Program Grant (300304). All views and opinions expressed herein are those of the authors and do not necessarily reflect those of our respective institutions or the Department of Veterans Affairs.

\section{References}

American Psychiatric Association. (1994). Diagnostic and statistical manual of menta disorders (4th ed.). Washington, DC: Author.

Angrist, J. D., Chen, S. H. \& Frandsen, B. R. (2010). Did Vietnam veterans get sicker in the 1990 ? The complicated effects of military service on self-reported health. Journal of Public Economics, 94, 824-837.

Blake, D. D., Weathers, F. W., Nagy, L. M., Kaloupek, D. G., Gusman, F. D., Charney, D. S., et al. (1995). The development of a clinician-administered PTSD scale. Journa of Traumatic Stress, 8, 75-90.

Blanchard, E. B., Hickling, E. J., Taylor, A. E., Loos, W. R. \& Gerardi, R. J. (1994). Psychological morbidity associated with motor vehicle accidents. Behaviour Research and Therapy, 32, 283-290.

Brown, T. A. \& McNiff, J. (2009). Specificity of autonomic arousal to DSM-IV panic disorder and posttraumatic stress disorder. Behaviour Research and Therapy, 47, 487-493.

Brown, T. A., Chorpita, B. F. \& Barlow, D. H. (1998). Structural relationships among dimensions of the DSM-IV anxiety and mood disorders and dimensions of negative affect, positive affect, and autonomic arousal.Journal of Abnormal Psychology 107, 179-192.

Chung, H. \& Breslau, N. (2008). The latent structure of post-traumatic stress disorder: tests of invariance by gender and trauma type. Psychological Medicine, 38(4), 563-573.

Clark, L. A. \& Watson, D. (1991). Tripartite model of anxiety and depression: Psychometric evidence and taxonomic implications. Journal of Abnormal Psychology, $100,316-336$

Cox, B. J., Clara, I. P. \& Enns, M. W. (2002). Posttraumatic stress disorder and the structure of common mental disorders. Depression and Anxiety, 15, 168-171.

Creamer, M., Burgess, P. \& McFarlane, A. C. (2001). Post-traumatic stress disorder: Findings from the Australian National Survey of Mental Health and Well-being. Psychological Medicine, 31, 1237-1247.

Creamer, M., Burgess, P. \& Pattison, P. (1992). Reaction to trauma: A cognitive processing model. Journal of Abnormal Psychology, 101(3), 452-459.

Del Piccolo, L., Saltini, A. \& Zimmerman, C. (1998). Which patients talk about stressfu events and social problems to the general practitioner? Psychological Medicine, 28, 1289-1299.

Elhai, J. D., Carvalho, L. F., Miguel, F. K., Palmieri, P. A., Primi, R. \& Frueh, B. C. (2011) Testing whether posttraumatic stress disorder and major depressive disorder are similar or unique constructs. Journal of Anxiety Disorders, 25, 404-410.

Elhai, J. D., Gray, M. J., Kashdan, T. B. \& Franklin, C. L. (2005). Which instruments are most commonly used to assess traumatic event exposure and posttraumatic effects? A survey of traumatic stress professionals. Journal of Traumatic Stress, $18,541-545$.

Flora, D. B. \& Curran, P. J. (2004). An empirical evaluation of alternative methods of estimation for confirmatory factor analysis with ordinal data. Psychological Methods, 9, 466-491. 
Foa, E. B., Steketee, G. \& Rothbaum, B. O. (1989). Behavioral/cognitive conceptualizations of post-traumatic stress disorder. Behavior Therapy, 20(2), 155-176.

Forbes, D., Fletcher, S., Parslow, P., Phelps, A. J., O’Donnell, M., Creamer, M., et al. Trauma at the hands of another: differences in the PTSD symptom profile following interpersonal compared with non-interpersonal trauma. Journal of Clinical Psychiatry, in press.

Forbes, D., Parslow, R., Creamer, M., O’Donnell, M., Bryant, R., McFarlane, A., et al. (2010). A longitudinal analysis of posttraumatic stress disorder symptoms and their relationship with fear and anxious-misery disorders: implications for DSMV. Journal of Affective Disorders, 127, 147-152.

Frueh, B. C., Elhai, J. D. \& Acierno, R. (2010). The future of posttraumatic stress disorder in the DSM. Psychological Injury and Law, 3, 260-270.

Frueh, B. C., Gold, P. B., Dammeyer, M., Pellegrin, K. P., Hamner, M. B., Johnson, M. R., et al. (2000). Differentiation of depression and PTSD symptoms in combat veterans. Depression and Anxiety, 11, 175-179.

Frueh, B. C., Grubaugh, A. L., Elhai, J. D. \& Buckley, T. C. (2007). U.S. Department of Veterans Affairs disability policies for PTSD: administrative trends and implications for treatment, rehabilitation, and research. American Journal of Public Health, 97, 2143-2145.

Grant, D. M., Beck, J. G., Marques, L., Palyo, S. A. \& Clapp, J. D. (2008). The structure of distress following trauma: Posttraumatic stress disorder, major depressive disorder, and generalised anxiety disorder. Journal of Abnormal Psychology, 117, $662-672$.

Gray, M. J., Elhai, J. D., Owen, J. R. \& Monroe, J. R. (2009). Psychometric properties of the Trauma Assessment for Adults. Depression and Anxiety, 26, 190-195.

Gros, D. F., Simms, L. J. \& Acierno, R. (2010). Specificity of posttraumatic stress disorder symptoms: an investigation of comorbidity between posttraumatic stress disorder symptoms and depression in treatment-seeking veterans. Journal of Nervous and Mental Disease, 198, 885-890.

Hoge, C. W., Castro, C. A., Messer, S. C., McGurk, D., Cotting, D. I. \& Koffman, R. L. (2004). Combat duty in Iraq and Afghanistan: mental health problems and barriers to care. New England Journal of Medicine, 351, 13-22.

Hu, L. \& Bentler, P. M. (1999). Cutoff criteria for fit indexes in covariance structure analysis: conventional criteria versus new alternatives. Structural Equation Modeling, 6, 1-55.

Keane, T. M. \& Kaloupek, D. G. (1982). Imaginal flooding in the treatment of a posttraumatic stress disorder. Journal of Consulting and Clinical Psychology, 50(1), $138-140$.

Kessler, R. C., Sonnega, A., Hughes, M. \& Nelson, C. B. (1995). Posttraumatic stress disorder in the national comorbidity survey. Archives of General Psychiatry, 52, 1048-1060.

King, D. W., Leskin, G. A., King, L. A. \& Weathers, F. W. (1998). Confirmatory factor analysis of the Clinician-Administered PTSD Scale: Evidence for the dimensionality of posttraumatic stress disorder. Psychological Assessment, 10, 90-96.

Krueger, R. F. (1999). The structure of common mental disorders. Archives of General Psychiatry, 56, 921-926.

Magruder, K. M., Frueh, B. C., Knapp, R. G., Davis, L., Hamner, M. B., Martin, R. H., et al. (2005). Prevalence of posttraumatic stress disorder in Veterans Affairs primary care clinics. General Hospital Psychiatry, 27, 169-179.

Marshall, G. N., Schell, T. L. \& Miles, J. N. V. (2010). All PTSD Symptoms Are Highly Associated With General Distress: Ramifications for the Dysphoria Symptom Cluster. Journal of Abnormal Psychology, 119(1), 126-135.
McNally, R. J. (2003). Progress and controversy in the study of posttraumatic stress disorder. Annual Review of Psychology, 54, 229-252.

Miller, M. W., Wolf, E. J., Harrington, K. M., Brown, T. A., Kaloupek, D. G. \& Keane, T. M. (2010). An evaluation of competing models for the structure of PTSD symptoms using external measures of comorbidity. Journal of Traumatic Stress, 23, 631-638. Muthén, B. O., \& Muthén, L. K. (2010). Mplus (Version 6). Los Angeles, CA.

Muthén, L. K. \& Muthén, B. O. (1998-2010). Mplus user's guide (6th ed.). Los Angeles, CA: Muthén \& Muthén.

Palmieri, P. A., Weathers, F. W., Difede, J. \& King, D. W. (2007). Confirmatory factor analysis of the PTSD Checklist and the Clinician-Administered PTSD Scale in disaster workers exposed to the World Trade Center Ground Zero. Journal of Abnormal Psychology, 116, 329-341.

Phelps, A., Forbes, D. \& Creamer, M. (2008). Understanding posttraumatic nightmares: An empirical and conceptual review. Clinical Psychology Review, 28, 338-355.

Pitman, R. K., Orr, S. P., Forgue, D. F., de Jong, J. T. \& Claiborn, J. (1987). Psychophysiologic assessment of posttraumatic stress disorder imagery in Vietnam combat veterans. Archives of General Psychiatry, 144(11), 970-975.

Resnick, H. S., Best, C. L., Kilpatrick, D. G., Freedy, J. R. \& Falsetti, S. A. (1993). Trauma Assessment for Adults: unpublished interview protocol. Charleston, SC: Medical University of South Carolina.

Rosen, G. M. \& Lilienfeld, S. O. (2008). Posttraumatic stress disorder: An empirical evaluation of core assumptions. Clinical Psychology Review, 28, 837-868.

Satorra, A. \& Bentler, P. M. (2001). A scaled difference chi-square test statistic for moment structure analysis. Psychometrika, 66, 507-514.

Sheehan, D. V., Lecrubier, Y., Sheehan, K. H., Amorim, P., Janavs, J., Weiller, E., et al. (1998). The Mini-International Neuropsychiatric Interview (MINI): the development and validation of a structured diagnostic psychiatric interview for DSM-IV and ICD-10. Journal of Clinical Psychiatry, 59(Suppl. 20), 22-33.

Sheehan, D. V., Lecrubier, Y., Sheehan, K. H., Janavs, J., Weiller, E., Keskiner, A., et al. (1997). The validity of the Mini International Neuropsychiatric Interview (MINI) according to the SCID-P and its reliability. European Psychiatry, 12, 232241.

Simms, L. J., Watson, D. \& Doebbeling, B. N. (2002). Confirmatory factor analyses of posttraumatic stress symptoms in deployed and nondeployed veterans of the Gulf War. Journal of Abnormal Psychology, 111, 637-647.

Slade, T. \& Watson, D. (2006). The structure of common DSM-IV and ICD-10 mental disorders in the Australian general population. Psychological Medicine, 36, 1593-1600.

Spitzer, R. L., First, M. B. \& Wakefield, J. C. (2007). Saving PTSD from itself in DSM-V. Journal of Anxiety Disorders, 21, 233-241.

Wang, P. S., Demler, O., Olfson, M., Pincus, H. A., Wells, K. B. \& Kessler, R. C. (2006) Changing profiles of service sectors used for mental health care in the United States. American Journal of Psychiatry, 163, 1187-1198.

Weathers, F. W., Keane, T. M. \& Davidson, J. R. (2001). Clinician-administered PTSD scale: a review of the first ten years of research. Depression and Anxiety, 13, 132-156.

Wirth, R. J. \& Edwards, M. C. (2007). Item factor analysis: current approaches and future directions. Psychological Methods, 12, 58-79.

Yu, C. Y. (2002). Evaluating cutoff criteria of model fit indices for latent variable models with binary and continuous outcomes. Unpublished Doctoral Dissertation, University of California, Los Angeles. 